\title{
A computational approach to modeling population differences
}

\author{
PATRICK CONLEY and CURT BURGESS \\ University of California, Riverside, Califormia
}

\begin{abstract}
Four experiments were conducted to determine whether the Hyperspace Analogue to Language (HAL) model of semantic memory could differentiate between two different populations. An analysis of the differences in densities (or average distances between word neighbors in semantic space) in HAL matrices-generated from text corpora derived from younger and older adults-confirmed that HAL was able to distinguish between the two age groups. This difference was again detected when structured interview data were used to build the corpora. A third experiment, designed to test the specificity of HAL in detecting differences between groups, did not detect any difference in the densities of the memory representations when older adults generated both the test corpora. The final experiment, conducted on the language of adults with Alzheimer's and normal adults, again demonstrated that HAL could discriminate between the two populations. These results suggest that HAL is capable of modeling, on the basis of changes in mean density, some of the differences between populations without modifying the model itself but, rather, by changing the text corpus from which the model creates its representations in semantic space.
\end{abstract}

The ability to adequately discriminate between populations is one of the foundations of psychological research. Detecting differences between groups allows researchers to isolate those variables that systematically change between the groups, permitting a better understanding of the processes and relationships between variables within the system under study. Even within the specific domain of cognitive psychology, the list of potential group contrasts is endless: younger and older adults, children and adults, adults with schizophrenia and normal adults, patients with Alzheimer's and normal controls, to name a few. Computational models have much to offer such research. First, computational models require the explicit operationalization of variables. Such operationalization itself often reveals confounds and hidden variables previously left unconsidered and allows for explicit testing of the contribution of each variable to the global performance of the model. Second, computational models often allow competing theories of differences between groups to be compared directly. Finally, computational approaches allow the modeling of processes that would be difficult to measure or even detect in actual human participants.

Computational models to date have primarily been used in specific domains, such as the effects of aging on cognition. For instance, Hannon and Hoyer (1994) demon-

This paper received the Castellan Award for best student submission at the 1999 conference of the Society for Computers in Psychology. The research was supported by an NSF Presidential Faculty Fellow Award SBR-9453406 to C.B. We thank Guila Glosser and Susan Kemper for providing us with the interview transcripts and subject data reported in this paper. Correspondence concerning this article should be addressed to P. Conley, Psychology Department, University of California, Riverside, CA 92521 (e-mail: patrick@cassandra.ucr.edu). strated a neural network model of the effects of aging on figure completion. In their experiment, Hannon and Hoyer employed three different methods to simulate aging in a neural network. The first method involved global changes in the strength between nodes, whereas the last two methods manipulated the ability of any two individual nodes to share information. The performance of the network after these manipulations did not support a global, diffuse model of cognitive slowing but, rather, specific localized deficits in figure completion processes. In this study, the benefit of implementing a computational model is clear. The neural network allows the direct comparison of several instantiations of competing theories of age-related deficits, while revealing and emphasizing the variables that have the greatest impact on network performance.

Hannon and Hoyer (1994) defined two problems in establishing a model of cognitive aging. The first problem is to choose an appropriate model for the behavior being examined and to describe the behavior of the model before an aging component has been implemented. Second, the behavior of the aging mechanism itself must be defined. This two-step experimental procedure is the accepted methodology in measuring the performance of computational models, not merely for aging effects, but across different populations in general. The unmodified model represents a baseline level of functioning (often analogous to normal adult processing), and the modified model instantiates some theoretical alteration in order to bring model output in line with human results for that population.

Another connectionist model of aging effects was implemented by Bowles (1994). In this study, the parameters of the model were adjusted to comply with the patterns exhibited by human participants. The methodology used in implementing the computational model in the 
Table 1

Semantic Word Neighborhood of Book in the HAL Model, With Distances in Riverside Context Units

\begin{tabular}{lc}
\hline Word & Distance \\
\hline story & 343 \\
game & 360 \\
movie & 368 \\
new & 368 \\
film & 382 \\
books & 384 \\
paper & 394 \\
recent & 395 \\
original & 396 \\
issue & 398 \\
series & 398 \\
word & 399 \\
\hline
\end{tabular}

Bowles study is similar to the process used in the Hannon and Hoyer (1994) study, in that a connectionist model is specifically altered to account for performance in human controls. In these studies, as in the general literature, no single computational model has been employed to demonstrate differences between a range of different populations without there being substantial modification to the model itself.

Our approach to modeling differences in populations (such as age) is somewhat different. Because our model is a high-dimensional memory model (the Hyperspace Analogue to Language, or HAL; Burgess \& Lund, 2000; Lund \& Burgess, 1996), in this paper, we examine whether different populations will have correspondingly different representations in memory and whether these differences in representation can be used to categorize and discriminate between populations. Unlike connectionist networks, changes in the HAL model are not implemented by altering the model itself but, rather, by providing different text sources - reflecting the differential experiences of the populations being compared-from which $\mathrm{HAL}$ bases its representations.

\section{THE HAL MODEL}

The HAL model of memory (Burgess \& Lund, 2000; Lund \& Burgess, 1996) has been used to investigate many aspects of memory and cognition. The HAL model produces semantic representations for words from a 320 million-word corpus of Usenet text. HAL generates weighted co-occurrence information by passing a 10 word window over the corpus of text and stores this cooccurrence information in a 70,000 $\times 70,000$ dimensional matrix. This matrix thus produces a vector for each of the 70,000 most frequent words in the corpus, and this vector contains the high-dimensional $(140,000)$ coordinates for plotting the word's location in semantic space. The contextual similarity between words is proportional to their proximity in this semantic space (see Table 1).

The HAL model has been used to investigate a broad range of cognitive phenomena, including priming effects, the dissociation between semantic and associative priming, category effects, and typicality effects (Burgess \&
Lund, 2000; Lund, Burgess, \& Audet, 1996). Although most work with HAL has been in the domain of traditional semantic and categorization research, the word vector representations encode meaning at a general level, not just at what is typically considered the semantic level. The strongest evidence for this is how HAL's representations encode grammatical categorical information (Burgess \& Lund, 1997). As a result, HAL's vector representations are best characterized as context vectors, rather than as semantic vectors, since they encode the contexts in which words appear and make use of this information in a variety of ways.

The capacity of the HAL model to categorize information has been thoroughly assessed. In one such study, we discovered that proper names had several interesting characteristics in the high-dimensional space (Burgess \& Conley, 1998). First, proper names clustered in their own area of semantic space, away from common nouns. If a proper name had name/noun ambiguity (such as bill), it would drift away from this proper name space toward the common noun space. However, more surprising than the segregation of proper names in high-dimensional space was their representation within that space. We found that proper names had denser representations than frequencymatched common nouns, a result that led us to wonder whether density might play a role in certain aspects of cognitive aging, since disproportionate difficulty with proper names is a deficit frequently reported by older adults (Cohen \& Burke, 1993). Density, in the HAL model, essentially represents how close together words are in the semantic space. The density of representations, or the average distances between a word and its neighbors in semantic space, is a general reflection of the semantic depth (or close relationships to related concepts) of any particular area of semantic space. Word densities in HAL have been shown to be sensitive to intraspace changes in representation, such as a difference between how proper names and common nouns are represented. However, the results of more recent research (Conley \& Burgess, in press) suggest that HAL word densities might also be sensitive to interspace changes in representation, or representational differences in memory between semantic spaces generated by different populations.

Priming in the HAL model is calculated by subtracting the distances in semantic space between the prime and a semantically related target and the same prime and a semantically unrelated target. The distance between prime and target is measured in Riverside Context Units (RCUs, a measure of semantic distance scaled for similarity to human reaction times). We found that the amount of semantic priming did not differ across matrices generated from a corpus of younger adults' text and a corresponding matrix derived from older adults' text (Conley \& Burgess, in press). However, we noticed that the absolute distances were smaller in the corpus for the older adults. Since this again raised the possibility that density of representation might differ between younger adults and older adults, we decided to test the ability of the HAL model to differentiate between the two. In the first experiment, we attempted 
best

series words best

words

books series

$\begin{array}{ccccc}\text { recent } & \text { game } & & \text { books } & \text { game } \\ \text { story } & & \text { recent } & \text { story } & \\ \text { BOOK } & \text { film } & & \text { BOOK } & \text { film }\end{array}$

movie new

movie new

\begin{tabular}{l} 
movie new \\
original $\quad$ paper \\
\hline Sparser Representations \\
Figure 1. A hypothetical illustration of sparse and dense word neighborhoods in semantic space, using ac- \\
tual HAL neighbors for the word book.
\end{tabular}

to determine whether the HAL model could discriminate between these two populations, younger and older adults, on the basis of the density of representations in highdimensional space.

\section{EXPERIMENT 1 \\ Differences Between Age Groups in the HAL Model}

Density, again, is a measure of the semantic depth of a certain region of semantic space, although proper names, with little semantic depth individually (i.e., proper names are essentially arbitrary in a contextual model), cluster tightly in their own dense area of semantic space (Burgess \& Conley, 1998). Semantic density has previously been found to be a reliable predictor of both priming and word recognition. (Buchanan, Burgess, \& Lund, 1996; Buchanan, Westbury, \& Burgess, 1999; Burgess \& Conley, 1998). Increasing the amount of text available to HAL increases density in the semantic representations. Adding text increases the number of weighted co-occurrences between words, which in turn increases the values stored in each word vector. This leads to denser representations in high-dimensional space, since words with more similar meanings come closer together as more instances of context similarity accrue. However, if the amount of text is held constant across corpora and there are no large differences between the numbers of unique words in each corpus, any mean density difference detected between two corpora should be due to a difference in the representations themselves (see Figure 1). In this experiment, we attempted to detect such density differences between age groups on the basis of the language generated by each of those groups.

\section{Method}

Materials and Procedure. In this experiment, the difference between age groups was operationalized by comparing memory matrices derived from two corpora-one generated by older adults, and one generated by younger adults, hereafter referred to as corpus age. The corpora were taken from the Usenet, and the authors were circumspect in selecting newsgroups with primarily younger or older adults as active participants in the group (an example of such a newsgroup was soc.retirement, included in the older corpus) and also in choosing newsgroups that were refereed or moderated to reduce inclusion of inappropriate text. The two text corpora from older adults (older corpus) and younger adults (younger corpus) each consisted of approximately 450,000 words. A total of 9,725 words were common to both the older and the younger corpora.

We analyzed the mean distances between 96 stimulus words from Conley and Burgess (in press) and the closest 10 neighbors to each of these stimulus words in semantic space for the matrices generated from the older and younger corpora. Word distance, again, is a measure of semantic relatedness; the more alike two word vectors are, the closer they will be in semantic space (Burgess \& Lund, 
2000; Lund \& Burgess, 1996). These mean distances between a word and its neighbors served as a measure of density in semantic space, and the means were compared across the two corpora.

\section{Results and Discussion}

A repeated measures $t$ test detected a difference in mean neighborhood distance between the older corpus ( $M=397$ RCUs) and the younger corpus [ $M=446$ RCUs; $t(95)=2.97, p<.01]$. This result demonstrates that the semantic space generated from the older corpus is, in fact, denser than the space derived from the younger corpus, even though both corpora contain exactly the same amount of text. Although we know that density increases as a function of the amount of text in the corpus, this cannot be the explanation for the results of this experiment, since text size was a constant across corpora. This finding supports the hypothesis that different populations have potentially different memory representations and that HAL is capable of detecting such differences when provided with a corpus generated by each group. The results of Experiment 1 are supported by the results of Experiments 2 and 3 and are generalized to yet another population in Experiment 4.

\section{EXPERIMENT 2 Modeling Age Differences in HAL With Interview Data}

A potential criticism of the methodology in Experiment 1 is that we cannot be sure that the density difference between corpora is due to age, because the anonymous nature of the Usenet prevents us from getting specific participant data. We must therefore assume that people who post to age-specific newsgroups are not misrepresenting their age. This is, admittedly, a large assumption, but one that we believe is justified and that we have taken pains to control for by selecting moderated groups with a minimum of off-topic posting.

Another possible confound is the wide range of topics that are discussed in the newsgroups; a narrower range of interests could result in more text's being associated with fewer topics, potentially resulting in denser representations. Furthermore, perhaps the stimuli used in Experiment 1 were not representative of the behavior of all of the words in the semantic space. To answer these criticisms, we took interview data with younger and older adults from Kemper, Kynette, Rash, and O'Brien (1989; Kemper, 1998). For these data, the ages of the participants were known, and the corpus material controlled by asking each participant specific questions in an interview setting. Two new memory matrices- the first derived from the interviews with younger adults and the second derived from the older adults - should provide further evidence regarding the nature of the representational differences detected between younger and older adults in Experiment 1.

\section{Method}

Participants. The participants for this experiment were 50 younger adults (18-28 years of age) and 50 older adults (60-
92 years of age). The younger adults participated for course credit, and the older adults were paid participants.

Materials and Procedure. In the interview, which Kemper et al. (1989) conducted individually, the participants were asked to describe the person that they most admired. This interview was then recorded and transcribed. Two corpora were created, the first consisting of interview transcripts with younger adults, the second consisting of interview material with older adults (again referred to here as corpus age). Each corpus contained 21,656 words of text (the older corpus was truncated by 6 words so that it would contain a number of words identical to that of the younger corpus). The list of shared vocabulary between the two corpora included 710 words.

We analyzed the mean distances between all 710 words in the shared vocabulary list and the 10 neighbors to each of these words that were closest in semantic space. Since each matrix consisted only of words that occurred in both text corpora, testing the shared vocabulary list signifies testing every word in the matrices. These means were measures of density in the semantic space, which were then compared across the two corpora.

\section{Results and Discussion}

We detected a difference between the density of the younger corpus ( $M=464 \mathrm{RCUs})$ and that of the older corpus $[M=445$ RCUs; $t(709)=3.10, p<.01]$. Again, the word densities from the matrix built from the text of older adults were greater than the corresponding densities derived from younger adults. This result provides further support that the HAL model is detecting a difference between the two corpora not on the basis of differences between topic breadth or unrepresentative experimental stimuli lists but, rather, on the basis of actual differences in representation between the semantic space generated by younger and older adults. These data also support the corpus sampling methodology of Experiment 1 ; in the present experiment, the ages of the participants were known and the topic controlled by specific questions, yet the results showed the same pattern as those reported in the previous experiment.

\section{EXPERIMENT 3 Comparing Corpora Generated by the Same Population}

Another potential criticism of the methodology outlined in the experiments above is that the HAL model might be so sensitive to density that it will detect density differences in any two corpora that it compares. If this were the case, small differences in the corpora might be sufficient to cause the differences in density that we demonstrated in the previous experiments, rather than the age-related differences that we hypothesize. To answer this concern, in this experiment, we compared two corpora, both derived from older adults. If age is indeed the factor that is leading to density differences between corpora, each of these new matrices should have the same level of density.

\section{Method}

The materials used in this experiment consisted of interview transcripts from older adults from two sources: the older adult interviews used in Experiment 2 (referred to as the first corpus) and 
older adult transcripts provided by Glosser and Deser (1990; the interview data provided by the authors make up the second corpus). The second corpus transcripts were derived from the interviews with 17 older adults with a mean age of 55.1 years. The second corpus contained 38,093 words, which was then truncated to 21,662 words, the exact size of the first corpus. The shared vocabulary list contained 749 words.

As in the previous experiment, 10 word neighborhoods were found for all words in the shared vocabulary list, and the mean distance for each neighborhood was computed. These mean densities, since they were derived for all the words in both matrices, represented a systematic measure of density for the entire semantic space.

\section{Results and Discussion}

No difference in density was detected between the first ( $M=443 \mathrm{RCUs}$ ) and second ( $M=445 \mathrm{RCUs}$ ) corpus $[t(748)=0.29]$. This result confirms that the HAL model is not so sensitive to density that it will detect a difference no matter what two corpora are used to generate its matrices. It is important to note that because the first and second corpora used in this experiment came from different researchers and different interview topics, the topics were much less related between corpora than was the case in Experiment 2, where both the older adults and the younger adult participants were asked identical interview questions. The results of this experiment and of Experiment 2 provide evidence that the representational differences between the younger and the older corpora in the previous experiments are due to age-related differences between the groups and not to the oversensitivity of the HAL model or to differences in the range of topic material in the text included in the corpora.

\section{EXPERIMENT 4 Alzheimer's-Related Differences in the HAL Model}

The results of Experiments 1 and 2 demonstrate that HAL is capable of discriminating between populations on the basis of the language of each population. The semantic space generated from the older corpus shows denser representations than does the semantic space generated from the younger corpus, despite the identical number of words in each corpus. However, is this density difference limited to comparisons between younger and older adults, or can the HAL model use density of representation to discriminate between still other populations? To determine the usefulness of density in the HAL model to distinguish population differences other than age, we obtained interview text from Alzheimer's patients and age-matched normal control participants. In this experiment, we examined whether the HAL model could similarly distinguish - in terms of word neighborhood density differences - between the interview texts of adults with Alzheimer's and those of normal older adults.

\section{Method}

Participants. Interview data from Glosser and Deser (1990) were provided to us by the authors for this experiment. The experimental participants consisted of 23 patients with probable Alzheimer's Disease, with a mean age of 64.3 years and a mean duration of symptoms of 4.9 years. The control participants group was made up of 41 normal control participants with a mean age of 55.1 years.

Materials and Procedure. To further test the ability of the HAL model to discriminate between populations, two more text corpora were constructed. The first corpus consisted of text transcribed from interviews with the adults with Alzheimer's disease. The second corpus was made up of text transcribed from interviews with the normal adult participants. Each corpus consisted of 19,073 words. The shared vocabulary list for the two corpora contained 877 words.

The mean distance in high-dimensional space for the 10 nearest neighbors was computed for each of the words in the shared vocabulary list of 877 words. Again, using the entire vocabulary list indicates that all of the words contained in both matrices were measured. The mean distances for each of these 877 word neighborhoods is a measure of density in semantic space in each of the memory matrices, and these means were then compared.

\section{Results and Discussion}

The density of semantic space was found to differ across the Alzheimer's ( $M=437$ RCUs) and the control $(M=452$ RCUs) corpora $[t(876)=3.657, p<.001]$. This result again demonstrates that the density metric is discriminating between memory matrices created from text sources generated by different populations. This experiment again tested every word in both matrices, demonstrating that the greater density of the matrix derived from text generated by adults with Alzheimer's was systematic throughout the entire matrix and was not due to sampling error.

Interestingly, we discovered that the representations in semantic space for older adults with Alzheimer's were even denser than the representations in semantic space for normal older adult controls. This result, as well as the results from the previous experiments and Conley and Burgess (in press), suggests that systematic changes in the density of representation might underlie or correspond with some of the difficulties associated with both normal aging and the onset of Alzheimer's disease.

\section{GENERAL DISCUSSION}

In this study, we have examined the effectiveness of the density metric from the HAL model of memory in discriminating between populations and, to some extent, the validity of such discriminations. Since distinguishing between populations is so vital to the field of psychology, many other computational models have attempted to do so in the past. The majority of these models, however, have a baseline level of performance that corresponds to normal adult behavior and have required that the model be modified by a human coder or programmer in order to account for differences between populations, such as the changes caused by aging. This is a limitation, since such modifications are usually implemented to alter the performance of the model until it closely compares with actual human subjects that belong to the population being examined. In these cases, the model is not so much detecting differences between populations as the programmer is distinguishing between groups and manipulating the model until it corresponds with human data. In the HAL model of memory, however, such manipulations 
are not necessary; there is no modification of the workings of the model. Rather, HAL can model certain aspects of population differences merely by generating its memory matrices from the language experience of the populations being compared. This process allows the HAL model to take advantage of the distinct memory representations of different groups without requiring specific alteration of the inner workings of the model.

The experiments reported in this study provide evidence that HAL can distinguish between older and younger adults on the basis of differences that mimic characteristics of the memory representations between the groups. Experiments 1, 2, and 3 demonstrated the ability of HAL to distinguish between age groups on the basis of density changes between the groups, with older adults consistently showing greater word densities in semantic space. The results of these experiments do not support the alternative explanations that differences in word frequency or the breadth of the topics discussed in the text corpora are the sources of the greater density reported in the representations of older adults. Furthermore, Experiment 4 demonstrates that the ability of the HAL model to discriminate between populations on the basis of density in semantic space is not limited solely to age differences.

Density in the HAL model has been shown in previous research to be a reliable predictor of priming and word recognition (Buchanan et al., 1996; Burgess \& Conley, 1998). Density also predicts naming and lexical decision time (Buchanan et al., 1999). This earlier research demonstrated that density was an important metric in measuring representational differences within a memory matrix. Now there is evidence that density in semantic space can also be used to distinguish between different matrices and, thus, between the populations from which those matrices were generated.

On the basis of these experimental results, we propose that the HAL model offers a unique computational contribution in distinguishing between populations, when actual language experience from the groups to be compared is provided, and that density of representation is fundamental to this process. Future research will address the ability of the model to generalize to other populations than the groups reported here and will more closely explore the role of semantic density in mental representations.

\section{REFERENCES}

Bowles, N. L. (1994). Age and rate of activation in semantic memory. Psychology \& Aging, 9, 414-429.

Buchanan, L., Burgess, C., \& Lund, K. (1996). Overcrowding in semantic neighborhoods: Modeling deep dyslexia. Brain \& Cognition, 30, $111-114$.

Buchanan, L., Westbury, C., \& Burgess, C. (1999). Use of density in predicting reaction time. Manuscript submitted for publication.

Burgess, C., \& Conley, P. (1998). Developing semantic representations for proper names. In M. A. Gernsbacher \& S. J. Derry (Eds.), Proceedings of the Cognitive Science Society (pp. 185-190). Hillsdale, $\mathrm{NJ}$ : Erlbaum.

BuRGESs, C., \& LuND, K. (1997). Modeling parsing constraints with high-dimensional context space. Language \& Cognitive Processes, 12, 177-210.

Burgess, C., \& LUND, K. (2000). The dynamics of meaning in memory. In E. Dietrich \& A. B. Markman (Eds.), Cognitive dynamics: Conceptual change in humans and machines (pp. 117-156). Hillsdale, NJ: Erlbaum.

Cohen, G., \& Burke, D. M. (1993). Memory for proper names: A review. Memory, 1, 249-263.

Conley, P., \& Burgess, C. (in press). Age effects in a computational model of memory. Brain \& Cognition.

Glosser, G., \& Deser, T. (1990). Patterns of discourse production among neurological patients with fluent language disorders. Brain \& Language, 40, 67-88.

HANNON, D. J., \& HoYer, W. J. (1994). Mechanisms of visual-cognitive aging: A neural network account. Aging \& Cognition, 1, 105-199.

KEMPER, S. (1998). [Language samples from younger and older adults]. Unpublished raw data.

Kemper, S., Kynette, D., Rash, S., \& O'Brien, K. (1989). Life-span changes to adults' language: Effects of memory and genre. Applied Psycholinguistics, 10, 49-66.

Lund, K., \& Burgess, C. (1996). Producing high-dimensional semantic spaces from lexical co-occurrence. Behavior Research Methods, Instruments, \& Computers, 28, 203-208.

LUND, K., BURGESS, C., \& AUDET, C. (1996). Dissociating semantic and associative word relationships using high-dimensional semantic space. In G. W. Cottrell (Ed.), Proceedings of the Cognitive Science Society (pp. 604-608). Hillsdale, NJ: Erlbaum.

(Manuscript received October 28, 1999; accepted for publication February 17, 2000.) 